\title{
PHASE CONTROL AND INTRA-PULSE PHASE COMPENSATION OF THE ADVANCED PHOTON SOURCE (APS) LINEAR ACCELERATOR*
}

\begin{abstract}
The submitted manuseript has been authored by a contractor of the U.S. Government by a contractor of the U.S. Government under contract No. Goverdingly, the U, S. Government retains a nonexclusive, royalty.free license to publish or reproduce the published form of this contribution, or allow others to do so, for U. S. Government purposes.
\end{abstract}

A. E. Grelick, N. Arnold, K. Ko, N. Sereno, and M. White, Argonne National Laboratory, 9700 South Cass Avenue, Argonne, Illinois 60439

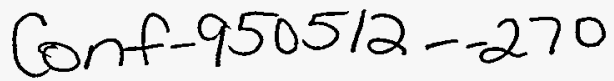

$=r_{n}$

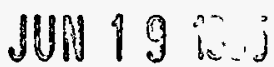

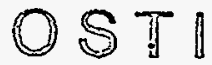

\section{Abstract}

Rf power for the APS linear accelerator is provided by five klystrons, each of which feeds one linac "sector," containing accelerating structures and SLED cavities. A VXI-based subsystem measures the phase of each sector of the linac with respect to a thermally stabilized reference line. The resulting information is used to control a linearized varactor phase shifter. Error correction is done by software, using operatorcontrollable parameters. A second phase shifter provides an intra-pulse correction to the phase of the klystron drive pulse. When the intra-pulse correction is applied, the resulting phase is flat to within $0.5^{\circ}$ after $2.5 \mu \mathrm{sec}$. A second correction, made after the PSK trigger to the SLED and during the filling of the accelerating structures, resulted in an energy gain of $5 \mathrm{MeV}$ from a single sector.

\section{INTRODUCTION}

The rf schematic for the APS linac is shown in Figure 1. Linac sectors are comprised of a klystron and associated accelerating structures.

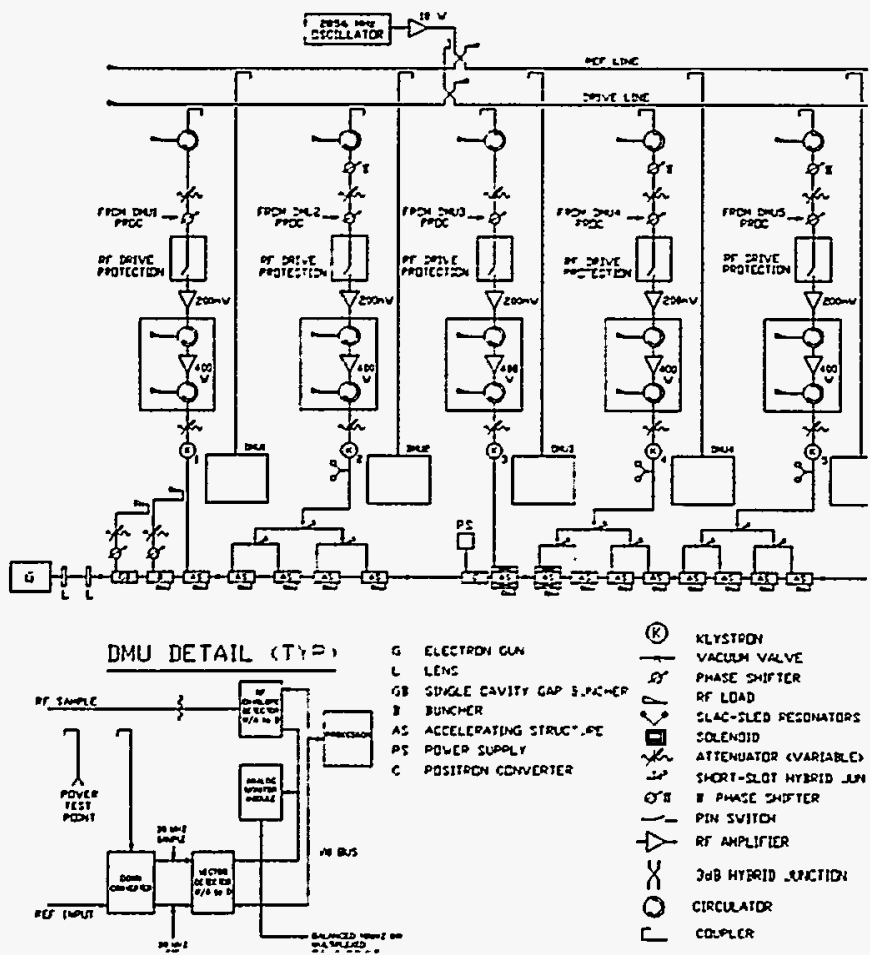

Figure 1: Schematic Diagram of the if system.

*Work supported by U.S. DOE Office of Basic Energy Sciences under Contract No. W-31-109-ENG-38.
The low-level rf system is principally housed in two separate cabinets for each sector: a VXI-based measuring system and a klystron drive system using a pulsed solid-state amplifier together with NIM modules and rfi-tight chassis.

\section{SYSTEM DESCRIPTION}

The entire system is driven from an ovenized, synthesized source and a 10-Watt GaAs FET amplifier. Both the drive and reference lines are driven from the amplifier output and are built into a single assembly which is temperature stabilized using water from one of the systems feeding the accelerating structures.

The VXI-based measuring system is based on Los Alamos National Laboratory (LANL) designed modules [1] with upgrades accomplished collaboratively by LANL and ANL. A common digital interface exists on all modules, while three types of on-board signal conditioning allow measurements of if amplitude, If phase, and beam position. Each channel digitizes a single measurement during each linac pulse, and the exact time of the sample can be adjusted by the operator. The modules for a sector are located in two C-size VXI mainframes, housed in a single 19-inch rack. MXI modules in slot zero of each of the two mainframes provide links between them.

The following VXI modules are used for if data collection and conversion:

Envelope Detector Modules (EDMs) provide eight channels of diode-detected signals. Linearized values for each possible raw output value from the analog-to-digital converter, interpolated from calibration of 88 points per channel, are stored in an EEPROM.

A Down Converter Module (DNM) driving a Vector Detector Module (VDM) produces two channels of I and Q data. The VDM operates at $20 \mathrm{MHz}$ and utilizes ovenized I and $\mathrm{Q}$ demodulators. Phase is calculated by software.

Analog-to-digital conversion in the EDM and VDM modules is done by a Datel SHM- 49 hybrid track/hold amplifier and an AD574 monolithic analog-to-digital converter, yielding a $10-\mathrm{MHz}$ bandwidth.

A different trigger timing system than the one used at LANL is used for most measurements in the APS linac and improves resolution and jitter by more than an order of magnitude. The upgraded LANL modules allow any of the VXI backplane triggers to be directly selected, or the LANL default triggering system can still be used. A VXI trigger module, designed at ANL, contains a set of eight-bit programmable delay lines which can be used to select sample time in increments of 5 


\section{DISCLAIMER}

Portions of this document may be illegible in electronic image products. Images are produced from the best available original document. 
nanoseconds. A separate delay line controls each of the two ECL triggers and eight TTL triggers on the VXI backplane.

Rf samples are available at the input (both forward and reflected) and output of each accelerating structure. Envelope detector channels have been provided for almost all of these signals. Multiplexed phase measurements are available for the forward power samples.

Key elements of the klystron drive chain before the pulsed amplifier are a bi-phase (PSK) modulator which is used to key the SLED [2] cavity assemblies in sectors L2, L4, and L5; a dual linearized phase shifter; a PIN diode switch controlled by a VSWR protection NIM module; and a preamplifier. The pulsed solid-state amplifier has a minimum power output of 400 watts and is followed by a motor-driven attenuator. After minimum attenuation and cable losses, drive power of at least 225 watts is available at the klystron input.

\section{PHASE CONTROL}

Phase is measured at the input to each sector (at the output of the klystron and SLED) with a single sample each pulse. Software computes a phase between $-180^{\circ}$ and $+180^{\circ}$, with a resolution of $0.01^{\circ}$, from measured $I$ and $Q$ data. The smaller in magnitude of $I$ and $Q$ is always used as the numerator in the computation to avoid losing precision near the sine wave maxima. Line stretcher type phase shifters are included at the reference inputs of each sector's phase measuring modules. These phase shifters are set so that the phase reading of each sector can be set to approximately $+90^{\circ}$ at maximum energy conditions for electrons. Therefore the readings for both electrons and positrons (roughly $-90^{\circ}$ ) will not usually be near the point of discontinuous readings located at \pm 180 degrees. It is also desirable that at this point phase is calculated as:

$$
\cos ^{-1}\left(\frac{I}{\left(I^{2}+Q^{2}\right)^{1 / 2}}\right)
$$

with I being near zero since the I channel has a lower noise level than the $Q$ channel.

An Allen-Bradley remote interface link from the VXIbased processor is used to implement phase control. Phase corrections are generally made when the measured phase error exceeds $2^{\circ}$ from a preset value chosen after the beam is optimized for energy, energy spread, and spot size and shape. Figure 2 is an example of the on-line window for phase control while operating with target in (i.e. with positrons).

\section{INTRAPULSE PHASE ERRORS AND SOLUTIONS}

Pulsed solid-state amplifiers are subject to several sources of phase error. One of the largest and most easily correctable is the variation in junction temperature over the pulse. Since the variation is exponential it can be corrected with an exponential predistorting phase shift. The correction technique used is the one aiready used on SLC [3] with two minor differences. Instead of combining the correction signal with the main phase control signal in an extremely linear phase shifter, a separate (but within a common package), commercially available linearized varactor phase shifter is used. Additionally, the range of possible correction time constants was increased from $5 \mu \mathrm{sec}$ maximum to $9 \mu \mathrm{sec}$ maximum. The need for longer time constants seems appropriate with the use of newer, larger junction transistors. With the correction optimized, the phase of the klystron drive pulse, after the initial $2.5 \mu \mathrm{sec}$, is flat to within $<0.5^{\circ}$. Noise on the I and Q outputs of the Vector Detector is the principal limiting factor. The klystron drive pulse is timed so that the initial $2.5 \mu \mathrm{sec}$ has an insignificant effect on the accelerated beam. In the SLEDed sectors, the drive pulse starts before the modulator trigger. In the other sectors, the drive pulse begins at least $3.3 \mu \mathrm{sec}$ before the electron gun trigger.

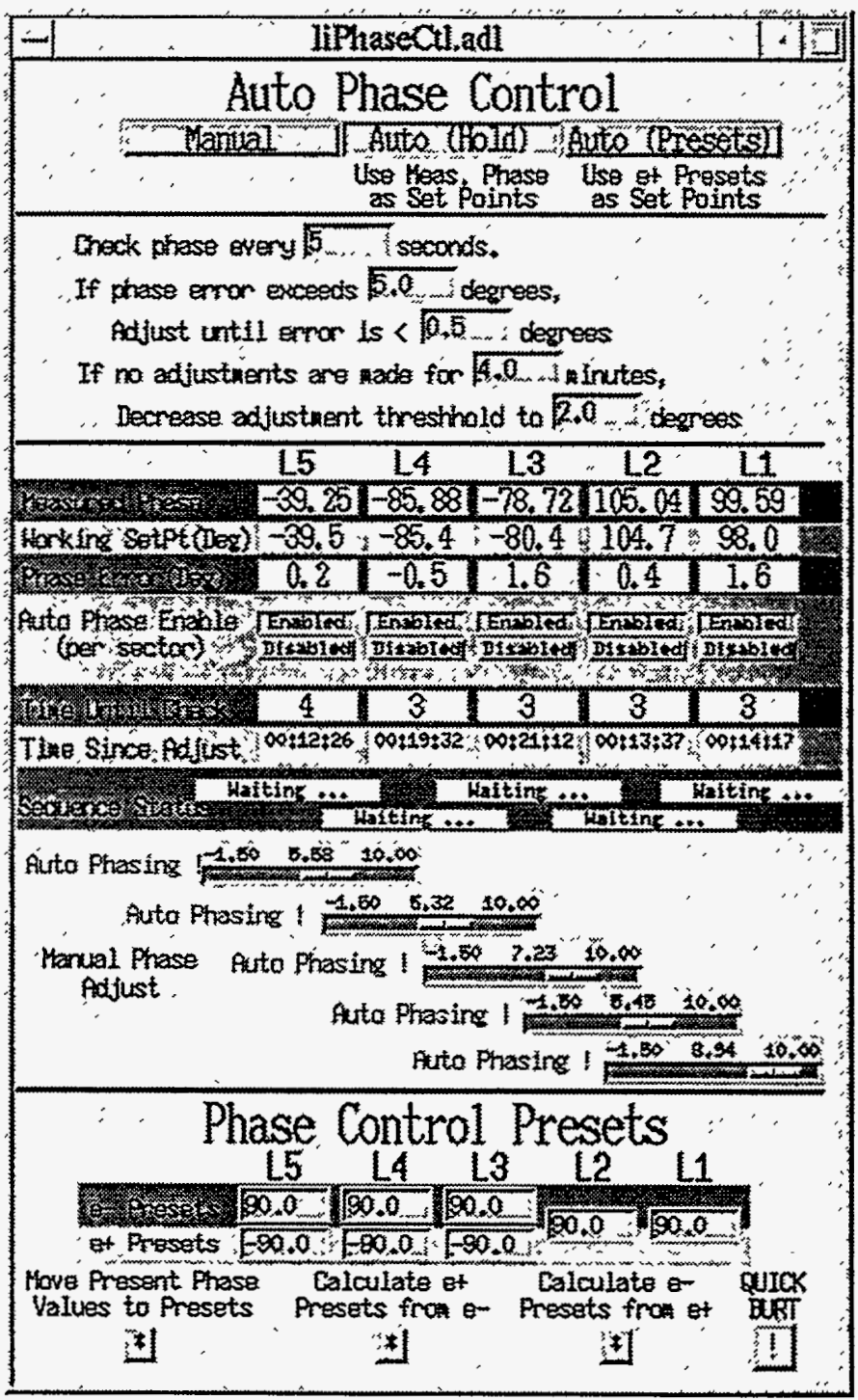

Figure 2: Autophase control screen during a positron run.

During commissioning, it was observed that less than ideal shape of the modulator pulse was limiting maximum available linac energy and that phase shifts over the pulse due to klystron phase pushing was a significant part of the limitation. In the pursuit of additional ways to increase maximum available linac energy, an experiment was performed on linac sector L2 (the best operating SLEDed sector at the time). 
An additional correcting signal was summed with the driver amplifier correction signal described above, during the part of the pulse after the PSK trigger, which corresponds to the accelerating structure fill time. A rectangular pulse of variable amplitude and polarity with width extending past the end of the modulator pulse was applied as part of the summed signal to the phase shifter. The limited response of the phase shifter integrated the leading edge of the pulse, giving the effect of adding a ramp phase shift component of variable slope which could be either positive or negative. The resulting slope was varied while watching a fluorescent screen following a calibrated analyzing magnet, giving an immediate readback of changes in beam energy. An energy increase of $5 \mathrm{MeV}$ was observed.

No decision has been reached on whether to implement such a correction. The results show an apparent possible 10$\mathrm{MeV}$ increase in positron energy by applying the additional correction in sectors L4 and L5. However, the magnitude of any possible increase in beam energy can be expected to tend towards zero as the ongoing activity to improve the modulators produces a pulse which more closely resembles the ideal [4].

\section{CONCLUSION}

This system has operated successfully for more than a year and has achieved repeatability to within its $\pm 2^{\circ}$ window which has been verified by its regular correlation with linac energy. It has also achieved a good and still improving level of reliability on-line. Basic performance has been at a level that has readily supported commissioning and operation while further refinements are still being pursued. More details of the linac's performance are given in [5].

\section{ACKNOWLEDGMENTS}

We would like to sincerely acknowledge the efforts of Messrs. D. Jefferson and J. Hawkins, whose efforts in support of the linac low-level systems were instrumental to the successful results.

\section{REFERENCES}

[1] S. P. Jachim, et al, "The Los Alamos VXI-Based Modular RF Control System," Proceedings of the 1993 Particle Accelerator Conference, Washington, DC., May 1993, pp 1154-1156, 1993.

[2] Z. D. Farkas, H.A. Hogg, G.A. Loew, and P.B. Wilson, "SLED: A Method of Doubling SLAC's Energy," SLACPUB-1453, June 1974.

[3] J. G. Judkins, J. E. Clendenin and H. D. Schwarz, "A Solid State High Power Amplifier for Driving the SLC Injector Klystron," IEEE Transactions on Nuclear Science, NS32, No. 5, pp. 2909-2911, Oct. 1985.

[4] T. J. Russell and A. Cours, "Klystron Modulator Operation and Upgrades for the APS Linac," these proceedings.

[5] M. White, et al., "Performance of the Advanced Photon Source (APS) Linear Accelerator," these proceedings. 